\title{
LARGE ALONG-TRACK BASELINE SAR-GMTI: FIRST RESULTS WITH THE TERRASAR-X/TANDEM-X SATELLITE CONSTELLATION
}

\author{
Stefan V. Baumgartner, Gerhard Krieger \\ Microwaves and Radar Institute, German Aerospace Center (DLR) \\ Muenchner Strasse 20, 82234 Wessling, GERMANY, Email: stefan.baumgartner@dlr.de
}

\begin{abstract}
In the paper first ground moving target indication (GMTI) and parameter estimation results obtained with the spaceborne TerraSAR-X/TanDEM-X satellite constellation are presented and discussed. For processing a dual-platform GMTI algorithm developed by the authors was used. This algorithm enables the estimation of the true geographical positions, the velocities and the headings of the detected targets with high accuracy. The algorithm is verified and evaluated using ground truth reference data.
\end{abstract}

Index Terms - Synthetic aperture radar, pulse Doppler radar, radar signal processing, road vehicle location

\section{INTRODUCTION}

Moving targets appear displaced from their actual positions in conventionally processed SAR images (cf. Fig. 1 top left) [1]. Therefore, the main objectives of almost any GMTI algorithm are the detection of the moving targets and the estimation of their true geographical positions. Furthermore, the velocities and headings of the targets are of interest and also should be estimated. However, parameter estimation is quite challenging, especially for GMTI algorithms which have to handle SAR data acquired with spaceborne sensors.

For a 'spaceborne' GMTI algorithm one of the most critical parameters to estimate is the moving target's broadside position or along-track displacement, respectively, which is directly related to the target's true geographical position. Having for instance only one single X-band satellite with two receiving antennas (e.g. one TerraSAR-X satellite) and computing the target's true position by exploiting the noisy and clutter disturbed along-track interferometric (ATI) phase, large position errors in the order of several hundreds of meters may occur [2]. Without incorporating a priori knowledge, e.g. the knowledge about the positions of the road axes in the SAR image, reliable parameter estimation often is not possible.

In [3] we have proposed a novel dual-platform SARGMTI algorithm which is not based on a priori knowledge. Since this algorithm does not rely on a road database, also vehicles moving on open land and on open water can be

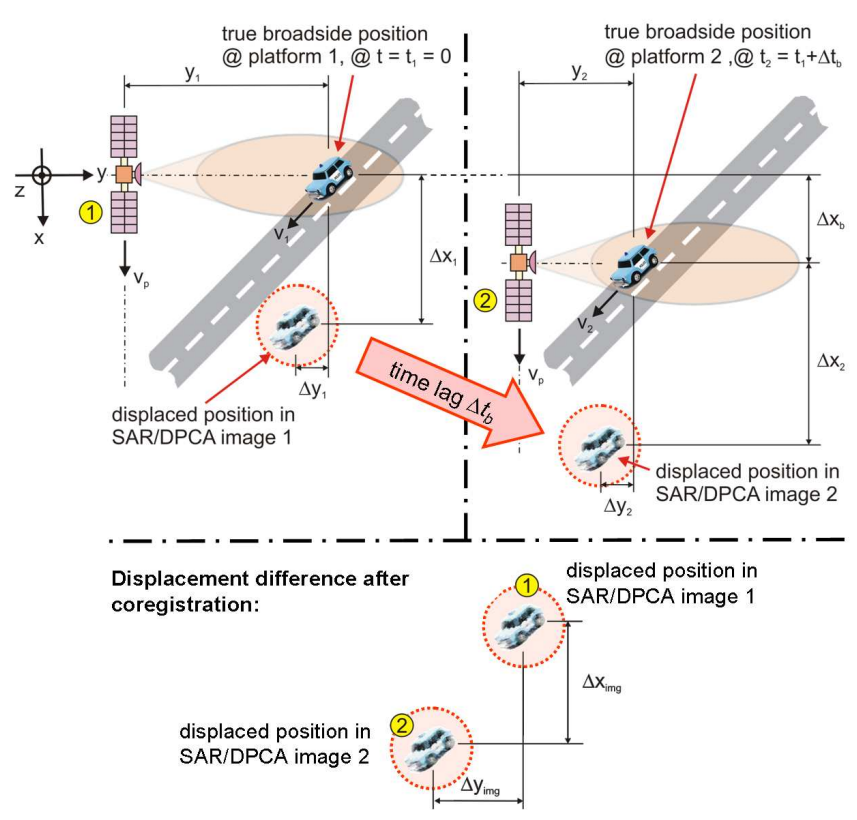

Fig. 1. Moving target displacements in the SAR images acquired with the first (top left) and second platform (top right). The displacement difference is shown at the bottom.

monitored. Theoretical analyses and simulations have predicted a high parameter estimation accuracy. For instance, the geographical position estimation error is only in the order of several meters instead of hundreds of meters using only a single SAR platform. However, since no real data were available when the GMTI algorithm was proposed in 2007, up to now it was not possible to verify the performance predictions.

Since June 2010 the TerraSAR-X/TanDEM-X satellite constellation is in orbit [4]. During the early commissioning phase the along-track baseline between both satellites was in the order of $20 \mathrm{~km}$, corresponding to a time lag $\Delta t_{\mathrm{b}}$ of approximately 2.5 seconds. This is just the time lag promising the best performance of the proposed GMTI algorithm [3]. Therefore, during the commissioning phase several GMTI data takes over different test sites have been acquired with the satellite constellation in pursuit monostatic mode, with the 

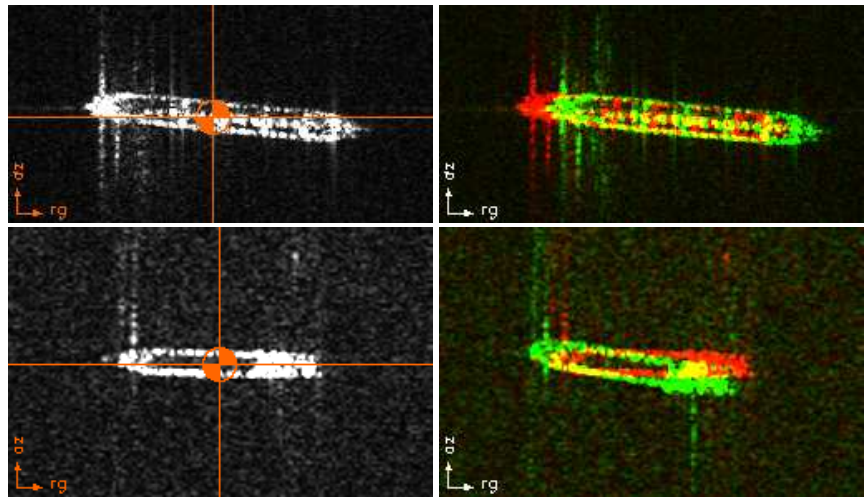

Fig. 2. TerraSAR-X images of two vessels (left col; the orange crosses mark the points used for georeferencing) and corresponding superpositions with TanDEM-X images (right col; TerraSAR-X image in red, TanDEM-X image in green).

aim to evaluate and verify the proposed GMTI algorithm.

In the following sections the principle of the GMTI algorithm is explained and the experimental results are presented and discussed.

\section{GMTI ALGORITHM PRINCIPLE}

The GMTI algorithm requires a large along-track baseline between both SAR platforms, so that the time lag $\Delta t_{\mathrm{b}}$ between the target observations is in the order of a few seconds. Owing to the large time lag, even slow targets move through several range and azimuth resolution cells between both observations. For that reason, the 'displaced' positions of the targets in both SAR images are different (cf. Fig. 1 bottom).

An example with real SAR data is shown in Fig. 2. In the right column the displacement differences clearly can be recognized. The time lag $\Delta t_{\mathrm{b}}$ between the acquisitions of TerraSAR-X (fore platform, image shown in red color) and TanDEM-X (aft platform, image in green) was approximately $2.5 \mathrm{~s}$. The vessel shown in the top row moved mainly from left to right (i.e. form near to far range) and the vessel in the lower row moved from right to left and additionally made a turn.

By performing a two-dimensional (2D) cross-correlation, the displacement differences in range as well as in along-track direction can be estimated. Prior to the 2D cross-correlation, clutter suppression and detection can be performed by using the displaced phase center antenna (DPCA) technique. Once the target is detected, also its Doppler slope can be estimated and the moving target images can be refocused for increasing the signal-to-clutter plus noise ratio (SCNR).

Knowing the radar parameters, the orbit-state-vectors of the platforms, the estimated Doppler slope and the estimated displacement differences, the true geographical position, the velocity and the heading of each detected moving target can

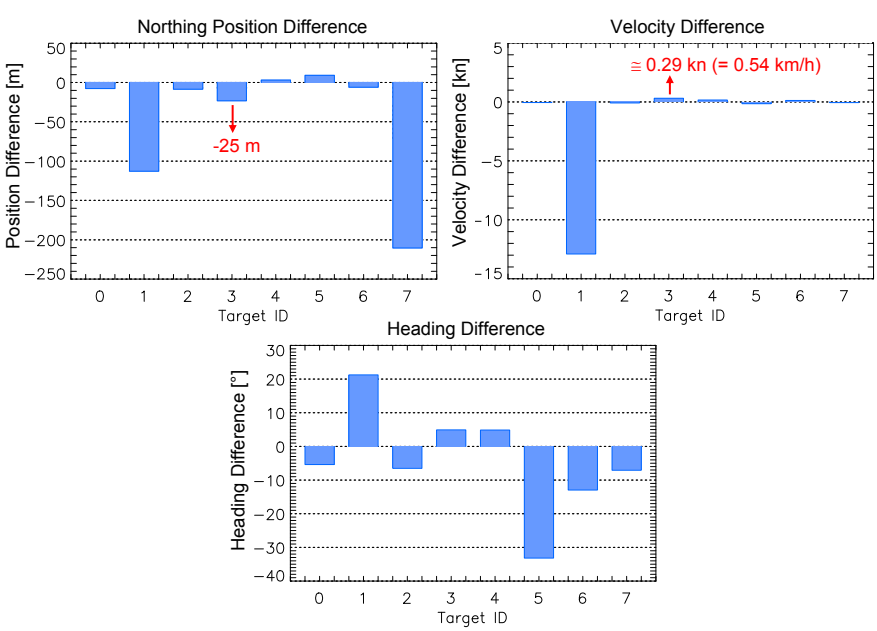

Fig. 3. Northing position differences (top left), velocity differences (top right) and heading differences (bottom) belonging to the Gibraltar data take.

be estimated with high accuracy. Even the acceleration can be estimated to a certain degree. A detailed derivation and explanation of the GMTI algorithm is given in [3] and should not be repeated here. However, it is necessary to extend the Cartesian acquisition geometry used in [3] to a spherical one for coping the approximately circular flight orbits of the SAR satellites.

\section{EXPERIMENTAL RESULTS}

In the following two subsections the GMTI results obtained from one maritime traffic and from one land traffic data take are presented and discussed.

\subsection{Maritime Traffic}

Maritime traffic was monitored in the Strait of Gibraltar. As ground truth automatic identification system (AIS) data were used. No clutter suppression was performed, only one single SAR image acquired with TerraSAR-X and one acquired with TanDEM-X were used for detection and parameter estimation.

In the SAR image large vessels appear as extended targets. For georeferencing the centroid of the area of the vessel image is used (cf. Fig. 2 left column). Note that the position of the centroid is different from the position of the AIS GPS receiver. Thus, the uncertainty of the computed position error depends on the vessel size. Possible turns of the vessels have to be considered, for instance by successively rotating the reference image patches containing the detected vessel images before the 2D cross-correlation is performed (cf. Fig. 2 bottom right). Without considering turns an accurate parameter estimation is not possible. 
The implemented dual-platform GMTI processor operates automatically and provides KML files, which can be viewed in Google Earth, as output. In Fig. 4 the GMTI results of the Gibraltar data take are shown. All eight vessels contained in the AIS database have been detected. By clicking on one of the vessel symbols a window containing all estimated vessel parameters appear, also the AIS information is displayed.

Top left of Fig. 3 shows the position differences, which were obtained by comparing the geographical positions estimated with the GMTI algorithm to the known AIS reference data. Owing to the used acquisition geometry and the major moving direction of the vessels along range (range direction differs only by $9.5^{\circ}$ from the UTM easting direction), the UTM northing position difference is directly related to the along-track position error. The uncertainty of the position error is in the order of half of the vessel width. The crosscorrelation result of the target with ID 1 was inaccurate (indicated by a small correlation coefficient), so that the position error with $-125 \mathrm{~m}$ is quite large. The time difference between the AIS data and the SAR data for target 7 was larger than $440 \mathrm{~s}$. It can be assumed that in this case the AIS position extrapolation (for which a constant vessel heading and velocity is assumed) is worse and that the true position error is much lower. Without target 1 and 7, the maximum position estimation error is $-25 \mathrm{~m}$, indicating that the parameter estimation is very accurate.

On the top right in Fig. 3 the velocity differences are shown. Again, the difference of the target with ID 1 is quite large owing to the bad correlation. All other values are below $0.29 \mathrm{kn}$ or $0.54 \mathrm{~km} / \mathrm{h}$, respectively, indicating a high velocity estimation accuracy.

The heading differences shown at the bottom of Fig. 3 are almost between \pm 5 and $\pm 10^{\circ}$, apart from the targets with ID 1 and ID 5. The target with ID 1 has a bad correlation coefficient and the target with ID 5 made a strong turn between both observations (cf. Fig. 2 bottom right). A low heading difference indicates that the velocity estimation in both dimensions (i.e. in range and in along-track direction) is very accurate.

\subsection{Land Traffic}

For monitoring land traffic one test site was the Interstate 15 (I15) in the north-east of Las Vegas. No ground truth data were available. Thus, the probability of detection cannot be estimated. However, since the geographical positions of the road axes can be obtained from a road database, the position estimation accuracy of the GMTI algorithm can be computed directly by measuring the residual offsets between the re-displaced targets and the road axes (cf. yellow arrow in Fig. 5). The velocity and heading accuracy can then be computed indirectly using the position estimation accuracy and the known direction of the road axes.

The dual-receive antenna mode, where the receiving an-

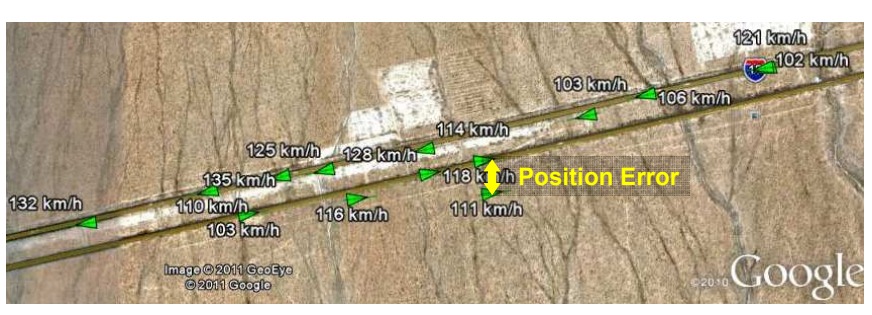

Fig. 5. GMTI result of the I15 data take as Google Earth overlay. The automatically detected road vehicles are depicted as colored triangles on their estimated geographical positions.
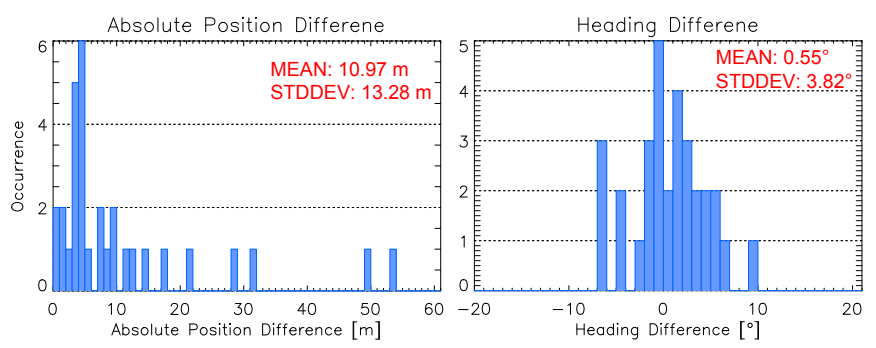

Fig. 6. Histograms of geographical position differences (left) and heading differences (right) with respect to the I15 road axes positions obtained from the OpenStreetMap database.

tenna of each satellite is split into two parts [4], was not available during GMTI data acquisition. Thus, instead of four SAR images in total only two SAR images have been acquired during each data take. Therefore, clutter suppression was performed by the DPCA technique, coherently subtracting the SAR image acquired with TanDEM-X from the image acquired with TerraSAR-X. Detection as well as parameter estimation were then performed by using only one single DPCA image.

The parameter estimation results of the $\mathrm{I} 15$ data take are shown in Fig. 6. False detections have been discarded manually since our interest is in the parameter estimation performance and not in the performance of the used detector. In total 31 potential moving vehicles with SCNR values between 10 and $23 \mathrm{~dB}$ remained for the performance investigations.

The accuracies of the estimated geographical vehicle positions, with a mean of $10.97 \mathrm{~m}$, are really impressive (cf. Fig. 6 left). To our knowledge no civilian SAR satellite system has ever reached these estimation accuracies before, particularly without the use of a priori knowledge. The mean value of $10.97 \mathrm{~m}$ corresponds to a velocity estimation accuracy of 0.57 $\mathrm{km} / \mathrm{h}$. Also the heading estimation accuracy is very accurate, as depicted on the right in Fig. 6.

\section{CONCLUSIONS}

The first experimental results presented in the paper indicate that the moving target parameter estimation accuracy of the 


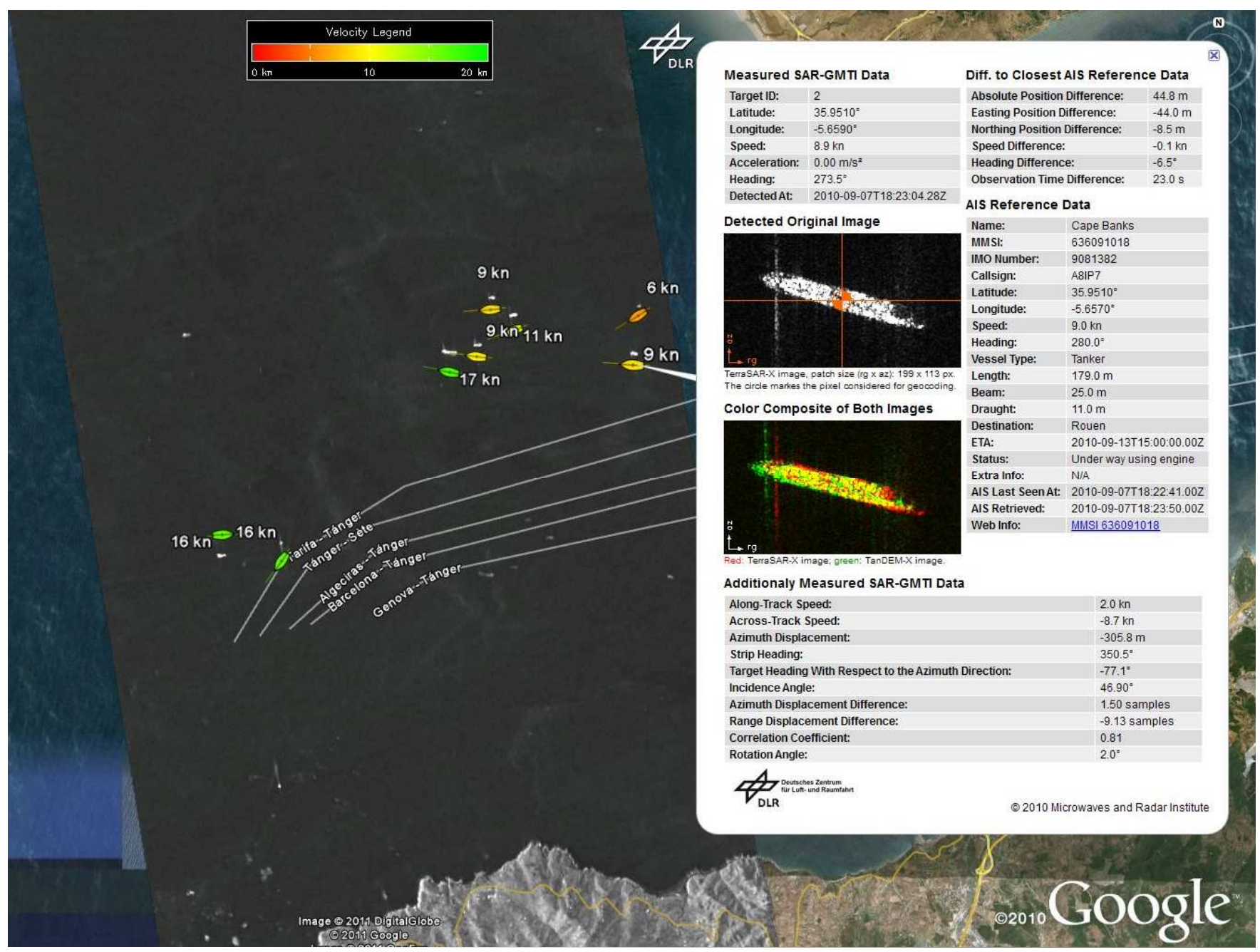

Fig. 4. Google Earth image of the Strait of Gibraltar overlaid with the KML file from the GMTI processor output. The color coded symbols (color is velocity dependent) mark the estimated 'true' geographical positions of the automatically detected vessels, also the displaced vessel images in white color are visible.

used GMTI algorithm reaches a superb performance. For land vehicles the position estimation accuracy is in the order of 11 $\mathrm{m}$ and for vessels better than $25 \mathrm{~m}$. Never before a civilian SAR satellite system has reached such an excellent moving target parameter estimation accuracy. In order to statistically confirm the first results presented in the paper, the remaining GMTI data takes still have to be evaluated.

\section{REFERENCES}

[1] R. K. Raney, "Synthetic aperture imaging radar and moving targets," IEEE Transactions on Aerospace and Electronic Systems, vol. AES-7, no. 3, pp. 499-505, May 1971.

[2] J. H. G. Ender, C. H. Gierull, and D. Cerutti-Maori, "Improved Space-Based Moving Target Indication via
Alternate Transmission and Receiver Switching," IEEE Transactions on Geoscience and Remote Sensing, vol. 46, no. 12, pp. 3960-3974, December 2008.

[3] S. V. Baumgartner, G. Krieger, and K.-H. Bethke, "A Large Along-Track Baseline Approach for Ground Moving Target Indication Using TanDEM-X," in International Radar Symposium (IRS), Cologne, Germany, September 2007.

[4] G. Krieger, A. Moreira, H. Fiedler, I. Hajnsek, M. Werner, M. Younis, and M. Zink, "TanDEM-X: A satellite formation for high-resolution SAR interferometry," IEEE Transactions on Geoscience and Remote Sensing, vol. 45, no. 11, pp. 3317-3341, 2007. 